\title{
Gesundheitsrisiken für Ärzte und Pfleger im OP: Massiver Handlungsbedarf beim Strahlenschutz
}

\author{
Beim Strahlenschutz im OP gibt es vielerorts Wissenslücken \\ aber auch Schlamperei beim Umgang mit den Vorschriften. \\ Die ZfOU bat Experten um Darstellung der wichtigsten Vor- \\ schriften - auch und gerade beim Einsatz von C-Bögen in OPs.
}

So schlecht hatten sich die Wissenschaftler das Ergebnis nicht vorgestellt. Über 500 Teilnehmer der jährlichen Fortbildungsveranstaltungen der AO Foundation in Davos befragte eine Gruppe um Dr. Alexander Joeris Ende 2015 nach ihrem Wissensstand und ihren Umgang mit den Vorschriften zum Thema Strahlenschutz (siehe das Interview ab Seite 323). Fazit: Der Grad der Besorgnis scheint fast umgekehrt proportional zur Bereitschaft, sich nach den Vorschriften zum eigenen Schutz zu richten. Über zwei Drittel der Teilnehmer machte sich zwar „mäßige bis sehr große“ Sorten um die eigene berufliche Strahlenbelastung. Fast $16 \%$ setzt einen Teil des eigenen Körpers häufig einem direkten Strahlengang aus. Aber nur 21\% trägt immer ein Dosimeter, die Hälfte nie, ein Drittel trägt keine Bleischürze, zwei Drittel trägt keinen Schilddrüsenschutz [1].

Eine „unnötig“ geringe Compliance konstatiert auch eine Gruppe um Thorsten Jentzsch vom Universitätsspital Zürich. Gut die Hälfte der 83 Teilnehmer in dieser

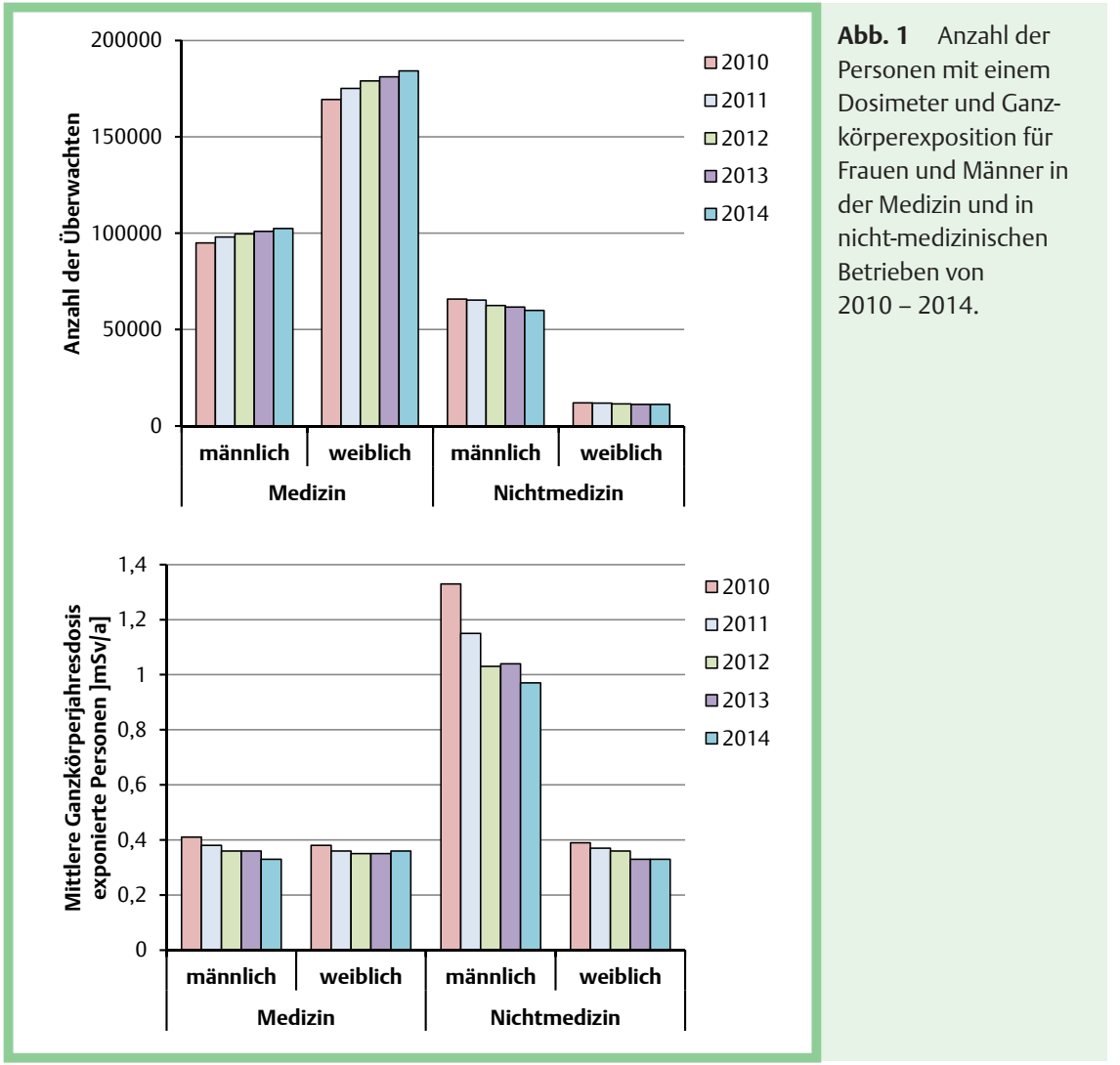

Umfrage, Mediziner wie Studenten und Pfleger in einem Traumazentrum und chirurgischer Abteilung eines Kinderkrankenhauses, hielt sich an die Vorschriften [2].

In den Kliniken fehle es an Kenntnissen und Schulungen zum Thema - bilanzieren Studienleiter Alexander Joeris von der AO Foundation wie auch der Unfallchirurg PD Dr. Michael Kraus von der der Donau-Ries Klinik in Donauwörth im ZfOU-Gespräch (siehe das Interview ab Seite 324).

Einige Eckpunkte der Spielregeln: Beim Einsatz von Röntgengeräten gilt die Röntgenverordnung. Kommen radioaktive Substanzen zum Einsatz, greift (obendrein) die Strahlenschutzverordnung. In Grundzügen sind die Spielregeln beider Verordnungen ähnlich.

$20 \mathrm{mSv}$ an effektiver Dosis an Strahlenbelastung im Jahr sind „erlaubt“ - es ist der „Grenzwert" für die berufliche Belastung, keineswegs ein Zielwert. Je weniger Belastung, desto besser ist die Grundregel im Strahlenschutz. Noch so kleine Dosen an ionisierender Strahlung erhöhen im Körper die Risiken auf Erkrankungen, biologisch gibt es keinen Grenzwert. Die „Berufslebensdosis“ darf 400 mSv nicht überschreiten.

Die effektive Ganzkörperdosis ist ein Kunstwert, bei dem auch berücksichtigt wird, wo Strahlen einwirken. Die Gefährlichkeit an Knochenmark oder Keimdrüsen ist höher und wird mit entsprechenden Wichtungsfaktoren höher bepunktet. Für einige weniger empfindlicher Organe sind hingegen höhere Werte einer lokalen Belastung als Organdosis noch gestattet, bei der Haut sind es $500 \mathrm{mSv}$.

Eine berufliche Belastung kommt quasi als Add-on zur natürlichen und zivilisatorischen Strahlenbelastung hinzu. 2,1 mSv kriegt jeder Bundesbürger im statistischen Mittel pro Jahr an natürlicher Strahlenbelastung $\mathrm{ab}$ - je nach Wohnort kann der Wert allerdings auf $10 \mathrm{mSv}$ und mehr schnellen, vor allem in Regionen mit hoher Radonbelastung [3]. Weitere 2,0 mSv an zivilisatorischer Belastung kommen hinzu - auch hier wiederum kann der Wert im Einzelfall deutlich steigen.

Wer in einem so genannten Kontrollbereich arbeitet - also zum Beispiel in einem $\mathrm{OP}$, in dem ein C-Bogen zum Einsatz kommt, muss zwingend bei der Arbeit ein 


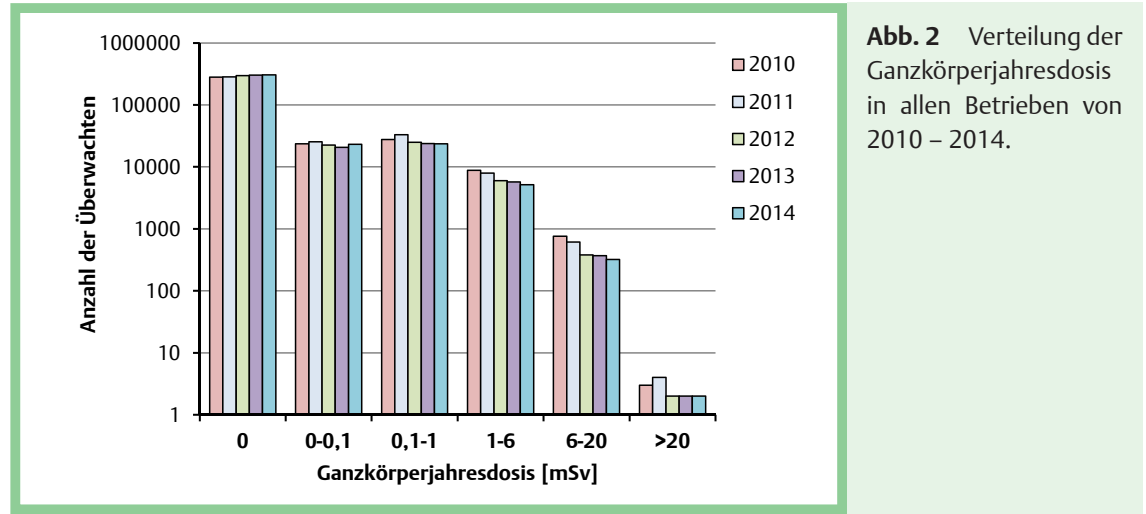

Dosimeter tragen, das monatlich ausgewertet wird (siehe das Interview ab S. 326). Über 350000 Beschäftigte tragen aktuell hierzulande ein Dosimeter. Das Bundesamt für Strahlenschutz führt eine Gesamtstatistik. Beschäftigte im Gesundheitswesen, alias „medizinischen Betrieben“ stellten danach 2014 mit 286822 das Gros der insgesamt 357660 „Überwachten Personen“ (o Abb. 1, [4]). Allein in Krankenhäusern trugen in 2014215690 Mitarbeiter Dosimeter [4]. Besser gesagt: Sollten ein Dosimeter tragen, denn etliche tragen es offenbar nicht regelmäßig oder gar kaum.

Trotz offenkundiger Mängel beim Beachten der Schutzvorschriften in der Praxis, eine gute Nachricht vorweg: Die Belastungen scheinen auf dem Rückzug. Die „mittlere Personendosis“ in allen medizinischen Betrieben, wie Krankenhaus, Arztpraxis nebst Sanatorium und Tierarztpraxis lag 2014 bei $0,35 \mathrm{mSv}$ im Jahr ( O Abb. 1, [4]) - das entspricht zum Vergleich in etwa der Strahlung, die ein Passagier bei 3-4 Flügen Frankfurt - New York hin und zurück abbekommt [5].

Nur 2 Personen hatten im Jahr 2014 eine Jahresdosis von über $20 \mathrm{mSv}$ und damit den Grenzwert überschritten ( $\triangle$ Abb.2, [4]). Auch im Bereich zwischen 6 und $20 \mathrm{mSv}$, so das BfS, seien die Werte in den letzten Jahren deutlich rückläufig. Mit 320 Personen in dieser Belastungsgruppe hätten sich die Werte seit 2010 um 60\% reduziert.

Dennoch - manch erfreulich niedriger Belastungswert in den Dosimetern kommt womöglich dadurch zustande, dass sein Inhaber das Gerät schlicht nicht anzieht, wie Experten argwöhnen. Solch Nichttragen wäre eigentlich ein Verstoß, der umgehend den Verantwortlichen im OP auf den Plan rufen muss, der die Fachkunde im Strahlenschutz hat und das Team anleitet, fordert Beatrix Kattinger von einer der zahlreichen Überwachungsbehörden auf Landesebene, sie arbeitet im Regierungspräsidium Stuttgart (siehe das Interview ab Seite 326). Zuständig in den Kliniken sind am Ende vor allem die so genannten Strahlenschutzbeauftragten. Apropos: Der Autor dieser Zeilen fand die Suche nach Ansprechpartnern just unter solch Strahlenschutzbeauftragten überraschend mühsam. Anfragen bei mehreren namhaften Unikliniken versandeten. Aber oh ja, man bemühe sich, die Vorschriften umzusetzen, aber Details, das führe rasch in einen „Dschungel an Vorschriften“ - so der Stoßseufzer eines Zuständigen, der dann doch nicht zitiert werden wollte.

\section{Literatur \\ 1 http://www.egms.de/static/en/meetings/ dkou2015/15dkou237.shtml \\ 2 http://www.egms.de/static/en/meetings/ dkou2015/15dkou235.shtml \\ 3 http://www.bfs.de/DE/themen/ion/um- welt/natuerliche-strahlenbelastung/natu- erliche-strahlenbelastung.html \\ 4 „Die berufliche Strahlenexposition in Deutschland 2013-2014, Download unter https://doris.bfs.de/jspui/ handle/urn: nbn: de: 0221-2015100213555 \\ 5 http://www.bfs.de/DE/themen/ion/um- welt/luft-boden/flug/flug.html}

\section{Weitere Informationen}

Strahlenschutzverordnung: http://www.gesetze-im-internet. de/strlschv_2001/

Röntgenschutzverordnung: http://www.gesetze-im-internet. de/r_v_1987/

\section{Bernhard Epping}

